EM

\title{
CIDADES MÉDIAS E INTEGRAÇÃO REGIONAL FRONTEIRIÇA
}

\author{
INTERMEDIATE CITIES AND REGIONAL BORDER INTEGRATION \\ CIUDADES INTERMEDIAS E INTEGRACIÓN REGIONAL FRONTERIZA ${ }^{1}$
}

\author{
Igor CATALÃO ${ }^{2}$ \\ João Henrique Zöehler LEMOS ${ }^{3}$
}

\begin{abstract}
Resumo: As cidades são nós articuladores da rede urbana e da divisão territorial do trabalho. Nos contextos de urbanização recente, expansão de fronteiras agrícolas e potencial para o desenvolvimento territorial, as cidades médias desempenham um particular papel de articulação e drenagem da renda regional. As discussões expostas neste artigo baseiam-se nas cidades de Posadas, Argentina - maior e mais importante cidade da província e importante centro articulador regional, inclusive à escala internacional - e, no contexto brasileiro, Chapecó e Passo Fundo - cidades médias que articulam uma ampla região onde predominam cidades pequenas. Ademais, estas últimas estão entre os 48 centros urbanos mais importantes de articulação do território brasileiro. Objetiva-se então analisar a potencial integração regional fronteiriça a partir das cidades médias mencionadas. Propõe-se discutir como a cooperação entre as cidades pode favorecer o desenvolvimento regional sem ignorar as contradições inerentes ao capitalismo. $\mathrm{O}$ eixo analítico reside na superação da perspectiva hierárquica da rede urbana, vislumbrando um desenvolvimento urbano-regional não metropolitano. O texto baseia-se em análise de dados, documentos e publicações acerca dos contextos mencionados e pretende contribuir para a análise integradora das cidades e regiões.
\end{abstract}

Palavras-Chave: Cidades médias; Desenvolvimento regional; Posadas; Chapecó; Passo Fundo.

Abstract: Cities are nodes that articulate the urban network and the territorial division of labour. In the context of recent urbanisation, expansion of agricultural frontiers and potential for territorial development, intermediate cities play a particular role in articulating and draining regional income. The discussions exposed in this article are based on the following cities: Posadas, Argentina, the largest and most important city in the province as well as an important regional articulation centre, including at international scale; and Chapecó and Passo Fundo, in Brazil, intermediate cities that articulate a wide region where small cities predominate. In addition, the latter cities count amongst the 48 most important urban centres in Brazilian territory. The aim of this article is to analyse the potential regional border integration from the perspective of the three mentioned intermediate cities. It is proposed to discuss how cooperation between cities can favour regional development without ignoring the contradictions inherent to capitalism. The analytical axis resides in overcoming the hierarchical perspective of the urban network, envisioning a non-metropolitan urban-regional development. The text is based on data, documents and publications analysis about the mentioned contexts and aims to contribute to the integrative analysis of cities and regions.

Keywords: Intermediate cities; Regional development; Posadas; Chapecó; Passo Fundo.

\footnotetext{
${ }^{1}$ La primera versión de este texto fue presentada el V Seminario Internacional de los Espacios de Frontera (V Geofrontera): territorialidades y sujetos transfronterizos, realizado en la Universidad Nacional de Misiones (UNaM), en Posadas, Argentina, los días 24 y 25 de octubre de 2019. El texto presenta reflexiones vinculadas al proyecto de investigación "Reestructuración urbana y diferenciación socioespacial en ciudades latinoamericanas", auspiciado por el CNPq. Esta versión del texto presenta pequeñas modificaciones frente a la versión presentada en el seminario.

${ }^{2}$ Docente investigador del Programa de Postgrado en Geografía de la Universidade Federal da Fronteira Sul (UFFS), igor.catalao@uffs.edu.br.

${ }^{3}$ Estudiante de maestría del Programa de Postgrado en Geografía de la Universidade Federal da Fronteira Sul (UFFS), becario CAPES, joao.zoehler@gmail.com.
} 
Resumen: Las ciudades son nudos articuladores de la red urbana y la división territorial del trabajo. En los contextos de urbanización reciente, expansión de fronteras agrícolas y potencial para el desarrollo territorial, las ciudades intermedias desempeñan un rol de articulación y drenaje de la renta regional. Las discusiones expuestas en este artículo se basan en las ciudades de Posadas, Argentina más grande e importante ciudad de la provincia y relevante centro articulador regional, incluso en escala internacional - y, el contexto brasileño, Chapecó y Passo Fundo - ciudades intermedias que articulan una amplia región en donde predominan ciudades pequeñas. Además, estas dos últimas están entre los 48 centros urbanos más importantes de articulación del territorio brasileño. El objetivo es analizar el potencial de integración regional fronteriza desde las ciudades intermedias mencionadas. Se propone discutir cómo la cooperación entre las ciudades puede favorecer el desarrollo regional sin perder de vista las contradicciones inherentes al capitalismo. El eje analítico reside en la superación de la perspectiva jerárquica de la red urbana hacia un desarrollo urbano-regional no metropolitano. La propuesta se basa en análisis de datos, documentos y publicaciones sobre los contextos tratados y pretende contribuir para el análisis integrado de las ciudades y regiones mencionadas.

Palabras Claves: Ciudades intermedias; Desarrollo regional; Posadas; Chapecó; Passo Fundo.

\section{Introducción}

El contexto actual de producción de ciudades tiene elementos que impactan de modos distintos los lugares y, por consiguiente, las variadas formas de organización de los centros urbanos. Cada uno de estos expresa funciones, roles y centralidades particulares en la red urbana en que se inserta. Se considera como una de las expresiones máxima de ello la integración entre las escalas local, nacional y supranacional yuxtapuestas, síntesis de la mundialización económica de la contemporaneidad, pero incluso de los efectos de proximidad en algunos contextos.

Las formas de dependencia entre los lugares se muestran intensificadas como nunca antes lo ha sido. Frente a ello, se construyen las interacciones que superan la tradicional lógica jerárquica de la articulación entre ciudades. En esa coyuntura, hay relaciones entre ciudades que se pueden nombrar heterárquicas de acuerdo a las abordadas en el trabajo de Catelan (2013). Estas interacciones corresponden al proceso de superación de la relación piramidal entre lugares, en el sentido señalado por Sposito (2006), aunque no sean propiamente lo opuesto a las relaciones convencionales. Se constituye, por lo tanto, una dialéctica jerarquía-heterarquía.

El capitalismo mundializado (CHESNAIS, 1996) necesita indiscriminadamente de nexos entre distintos lugares, especialmente en el actual contexto de flexibilización de la producción, grandemente pautado por el consumo generalizado de bienes y servicios a despecho del modelo industrial predominante en otros momentos históricos. El libre tránsito 
de capitales en los territorios nacionales conforma una nueva realidad contemporánea, que es discutida por Soja (1993) cuándo nos presenta un amplio panorama geo-histórico del capitalismo mundial. Se trata de un extenso escenario de reestructuración productiva en los niveles urbanos y regionales, en busca de una reubicación de las fuentes de extracción de lucros sectoriales. Esta dinámica tiene respaldo en una frenética movilidad del capital, y es sedienta por las rupturas de las jerarquías establecidas, en alianza de amplias debilitaciones de los controles políticos - pero requiere la manutención del Estado como ente financiador para las corporaciones - que impulsan los ciclos de desarrollo desigual en los planes verticales y horizontales del espacio mundial (OSORIO, 2004; SANTOS, 2000; SMITH, 1988; SOJA, 1993).

En ese contexto, se reafirma la necesidad de búsqueda por otros caminos al desarrollo local y regional, principalmente en un período en que el campo de conflictos institucionales y políticos se muestra acelerado frente a las necesidades provocadas por las crisis del capitalismo, marcados por "cambios radicales continuos que han marcado las tres décadas pasadas del siglo XX” (SOJA, 2005, p. 68). Ese desencaje del giro de producción de beneficios en una escala no vista antes requiere, a partir de perspectivas renovadas, otras visiones sobre los potenciales de ciudades y regiones. Santos (2013) ya apuntaba hacia las ciudades regionales como contextos favorables a la planificación hecha de manera más próxima de las demandas locales, bajo la potencial condición de enfrentamiento a las verticalidades, que son verdaderos imperativos llenos de contradicciones, marcas fundantes de nuestro tiempo.

Este texto pretende establecer elementos para la reflexión de nuevas posibilidades de integración en contextos fronterizos de Sudamérica, abarcando ciudades intermedias ${ }^{4}$ de las redes urbanas y otros elementos que no la especializada producción económica, ampliamente dependiente de interacciones transcontinentales vinculadas a la exportación. Desde ahí, se trata de una lectura que parte de la ciudad como realidad local favorable a nuevos horizontes de un desarrollo que se diseña por las necesidades más equitativas, justas y productivas desde el punto de vista social.

El análisis se base en exploraciones sobre la ciudad argentina de Posadas, capital de la provincia de Misiones, y dos ciudades en el Sur brasileño: Chapecó, ubicada en el oeste del

\footnotetext{
${ }^{4}$ El debate sobre las ciudades medias/intermedias es amplio y controvertido y ha recibido mirada especial en Brasil, Chile, España y Francia. La manera como entendemos la noción será presentada en el texto más adelante. Utilizamos en el texto la expresión en castellano "ciudad intermedia" acompañando Bellet y Llop (2004) y Bellet y Sposito (2009), entre otros.
} 
estado de Santa Catarina, y Passo Fundo, situada en el noroeste del estado de Rio Grande do Sul. En la Figura 1 es posible observar la ubicación de estas ciudades tal como la gran densidad de divisiones político-administrativas. Se destaca una profusión de municipios que podría hacernos cuestionar las gestiones públicas en los niveles locales y regionales, basándonos en una red de ciudades articuladas con los principales centros urbanos, como los que estamos proponiendo discutir aquí.

Figura 1 - Posición geográfica de las ciudades de Posadas/Misiones/AR, Chapecó/SC/BR y

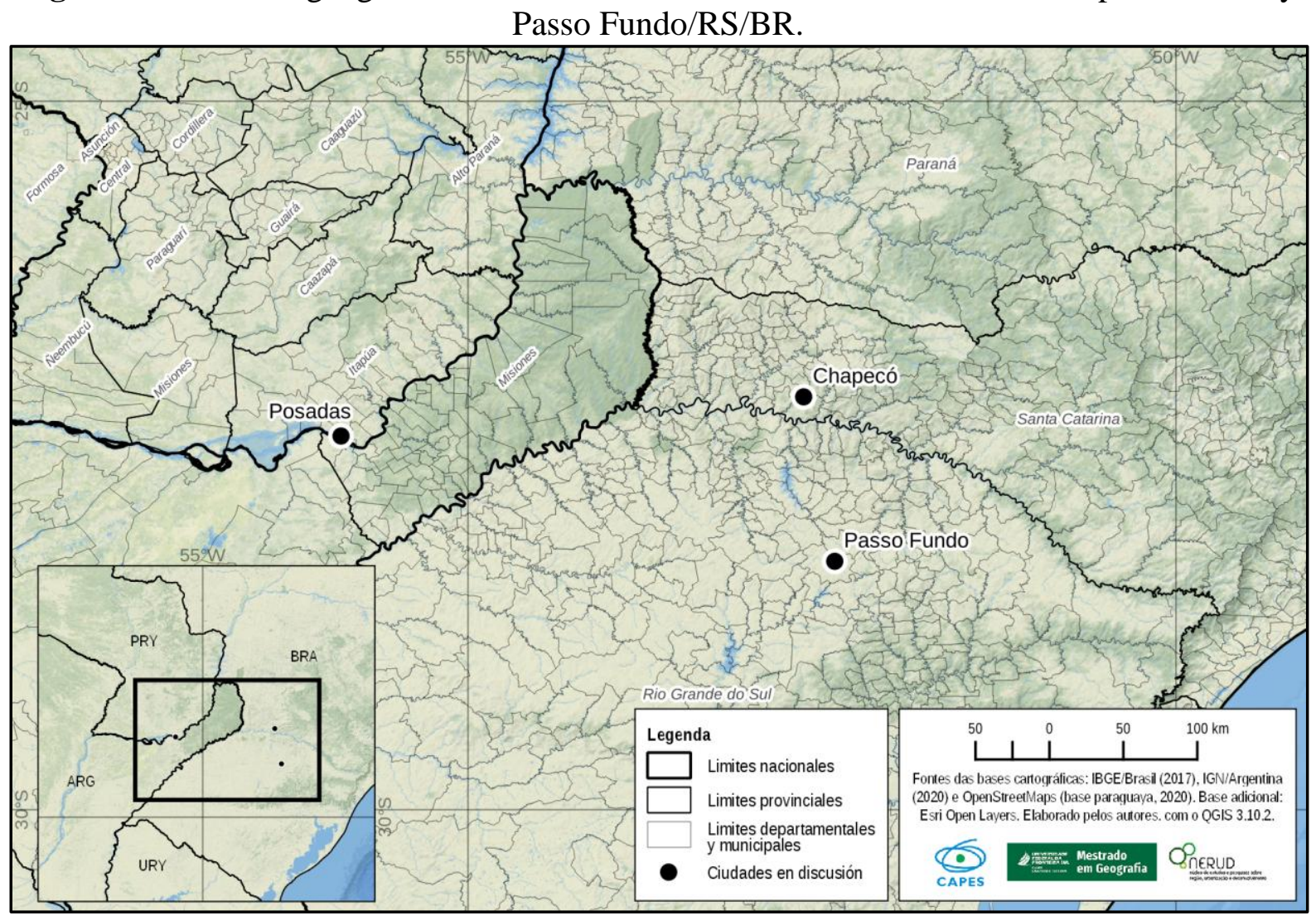

Fuente: Elaborado por João Henrique Zoehler Lemos.

En esos tres centros urbanos se identifican roles relevantes de las redes urbanas de que forman parte, además del hecho de que han demostrado altas tasas de crecimiento poblacional y económico en las últimas décadas. El crecimiento multisectorial debe, sobre todo, convertirse en mejores condiciones para el desarrollo socioespacial, bajo una visión que recupere el principio de las interacciones sociales en cuanto principios dialecticos de avanzos en las condiciones justas de vida, de acceso a bienes públicos. 


\section{Ciudades intermedias y escalas de la urbanización}

El cuadro actual de la producción del espacio mundial está fuertemente marcado por flujos de diferentes calidades que, en la busca incesante de plusvalía, superan cualquier tipo de frontera en niveles amplios. Ese contexto se hace fecundo en dimensiones de análisis sobre uno de sus productos más significativamente provocadores: las ciudades y sus dinámicas contemporáneas.

En la estera de la producción de ciudades que impactan en escalas locales, regionales y nacionales, se encuentran las ciudades intermedias, entendidas como las que se constituyen a partir de una muy específica "combinação de tamanho demográfico, funções urbanas e a organização de seu espaço intraurbano" (CORRÊA, 2007, p. 24-25).

Según las contribuciones de Brandão (2017), las ciudades intermedias son lugares que concentran equipamientos de alcance e impacto regionales, comprensión que dialoga con las reflexiones de Corrêa (2007) sobre el rol de intermediación/interacción con las regiones del entorno. Mientras la intermediación puede ser observada desde la lógica clásica de relación jerárquica ya mencionada, la interacción propuesta se dirige a una influencia que promueve relaciones propias. Se constituye como un subconjunto de flujos multicualitativos producidos desde las propias ciudades intermedias y demás ciudades de su región (CATELAN, 2013; BRANDÃO, 2017).

Aquí presentamos las tres ciudades analizadas como planos empíricos para la reflexión teórico-conceptual sobre las potencialidades regionales de las ciudades intermedias. En ese sentido, tanto Posadas como Chapecó o Passo Fundo demuestran elementos que nos llevan a interpretarlas como lugares donde el proceso de urbanización se muestra reforzado, con intensos cambios en las cuestiones inmobiliarias, industriales y de servicios especializados (MICHELINI; DAVIES, 2009; SOBARZO, 2010; MATIELLO et al., 2016).

Pensar en una conexión entre ciudades en escalas simultáneamente regionales (Latinoamérica) e internacionales (Argentina-Brasil), como para potenciar las articulaciones entre las tres ciudades mencionadas, se presenta como una perspectiva frente a la fragmentación del poder nacional en distintos contextos, como se observa desde hace mucho tiempo en países sudamericanos (HAESBAERT, 2001). Ello produce una serie de tensiones que expresan lo "no común" de la contemporaneidad, en donde las diversas crisis existentes en las relaciones capitalistas entre Estados nacionales se intensifican de manera no vista antes, basada en un conjunto de cuestiones que abarcan: las crisis de inmigración y desplazamiento 
de refugiados; las nuevas y precarias perspectivas de trabajo y empleo; el refuncionalizado espacio público (en sentido amplio); y la apropiación económico-financiera del Estado por entes privados etc. (DARDOT; LAVAL, 2017).

Aunque se considere, en este trabajo, que los equipamientos de uso colectivo existentes en las ciudades analizadas, principalmente los públicos, deberían estar bien distribuidos en los diversos lugares — como un horizonte ideal para reducir desplazamientos —, se identifica, de modo más objetivo, una posibilidad de integración regional fronteriza emergente. Por ende, se avanza en una perspectiva analítica señalada por un (contra) desarrollo, tal como propuesto en Catalão, Magrini y Lindo (2019), es decir, la búsqueda de alternativas al desarrollo convencional, especialmente en países periféricos de la economía capitalista (Wettstein, 1992). En otras palabras, se trata de valorar, por un lado, los beneficios del desarrollo capitalista, principalmente los materializados en infraestructuras de todos tipos, y avanzar, por otro lado, para romper la estrategia hegemónica del desarrollo para producir desigualdades y generar escasez de todos órdenes a las mayorías, como hemos visto de modo bastante claro en contextos del Sur global (MABIN, 2015).

Las varias formaciones socioespaciales en escalas regionales que conforman las centralidades de Posadas, Passo Fundo y Chapecó - ciudades que son nudos importantes en las redes urbanas de que forman parte - hoy se caracterizan por las transformaciones recientes en la producción de sus espacios, relacionándose más o menos intensamente con la ampliación del éxodo rural, la tecnificación del campo que la genera y la concentración de población en centros de más grande relevancia regional (MAIDANA; MILLÁN, 2009; MICHELINI; DAVIES, 2009; PORTO-GONÇALVES, 2017).

En un cuadro más general, Posadas, capital de Misiones y ciudad fronteriza a la ciudad paraguaya de Encarnación, tenía en 2010 una población de 275,9 mil habitantes según el censo demográfico nacional argentino, habiendo presentado un crecimiento de población bastante significativo en las últimas dos décadas. Se nota, por ejemplo, un aumento de más de 70 mil personas en relación el censo de 1991, es decir, un incremento considerable de migrantes, principalmente provenientes de las ciudades cercanas, en función de cambios en la estructura productiva regional, de la inauguración de la Usina Hidroeléctrica de Yacyretá y de políticas de integración y acuerdos comerciales con el Paraguay (MICHELINI e DAVIES, 2009). 
Tras los años de reestructuración ${ }^{5}$, el espacio urbano de Posadas se caracteriza por importantes cambios, incluso direccionados por una política estatal de remodelación urbana que, según Michelini y Davies (2009), fue excluyente, elitista y no contó con la participación de los ciudadanos. Los autores comentan que hay un interés de producir un discurso de modernización en la ciudad que es central en la región del Alto Río Paraná. En el transcurso de las acciones de recualificación de los espacios de la ciudad, el pensamiento particular (y privatista) acerca de una supuesta modernización se hace colectivo. Maricato (2011, p. 165) señala que la "representação da cidade encobre a realidade científica" a través de una muy fuerte publicidad que encubre los aspectos perversos. Se lo puede notar por la expansión de servicios especializados y del propio tejido urbano de modo a dispersar segmentos de la población y producir no sólo concentración de renta, sino también segregación residencial.

Chapecó y Passo Fundo, ubicadas respectivamente en los estados brasileros de Santa Catarina y Rio Grande do Sul, son centrales en una región cuya formación socioespacial difiere en relación a la región argentina vecina. Chapecó es el centro de una región caracterizada por compleja producción agropecuaria industrializada. Es la ciudad más importante y centro de gestión del territorio, responsable por drenar la renta regional. El Instituto Brasileiro de Geografia e Estatística (IBGE) estima que, en 2019, la ciudad haya alcanzado 220,4 mil habitantes, es decir, un potencial aumento en relación al censo de 2010, cuando su población era de 183,5 mil habitantes.

La Región Geográfica Intermedia de Chapecó, como la nombra el IBGE, corresponde a la mayor parte de la porción oeste de Santa Catarina, cuya densidad municipal es elevada, formada por una centena de municipios. Las ciudades de esta región se han constituido especialmente bajo una forma muy particular de reapropiación del territorio basada en una estructura de pequeñas propiedades (MATIELLO et al, 2016). Esos elementos, entre otros, producen una intensa y compleja división territorial del trabajo que es bastante jerarquizada y se apoya en amplia movilidad de población - sobre todo laboral - y existencia de varios centros urbanos regionales, aunque ninguno de ellos de igual importancia con Chapecó. Esta ciudad ha demostrado una ampliación de servicios especializados de salud, educación y consumo, especialmente en la última década, en sintonía con lo ocurrido en todo el territorio

\footnotetext{
${ }^{5}$ Entendemos reestructuración en el sentido que le da Soja (1993), o sea, de adaptaciones a los cambios socioculturales y económicos del capitalismo frente a las crisis generadas. No se trata, por ende, de una ruptura completa del modo de producción (o civilizatorio), sino más bien de una frenada y cambio de dirección. En las ciudades y redes urbanas, esta reestructuración suele tener características propias por lo que se le nombra urbana.
} 
brasileño al largo de la primera década y media de este siglo (CATALÃO; MAGRINI; LINDO, 2017).

Passo Fundo, a su vez, tiene una situación geográfica semejante a las otras dos ciudades ya mencionadas. Para Sobarzo (2010), se trata de un relevante y el más complejo centro urbano del norte de Rio Grande do Sul, razón por la que la Región Geográfica Intermedia de Passo Fundo definida por el IBGE lleva su nombre. Su formación es bastante antigua, pero hoy tiene paralelos con algunos elementos más recientes de la propia formación de Posadas. La red de ciudades que organiza forma una región en la que, de un lado, se destacan grandes propiedades rurales y, de otro, una división territorial en forma de mosaicos de pequeñas propiedades. Ello refuerza una serie de actividades existentes en el espacio regional, especialmente las actividades de enseñanza superior, salud de alta y media complexidad, bien como las tecnificadas prácticas agropecuarias más recientes.

Tal como Chapecó y Posadas, Passo Fundo, con sus estimados 203,3 mil habitantes en 2019, concentra servicios de salud y educación y es un importante cruzamiento de carreteras. Esas tres ciudades (situadas en el mapa de la Figura 1) son las que, en una amplia región transfronteriza, reúnen un conjunto de característica que les confieren centralidades de cualidades distintas, pero potentes para articular las ciudades de sus entornos en términos de producción, circulación y consumo.

En la red urbana brasileña, Chapecó y Passo Fundo están en una condición importante entre las ciudades del Sur del país. Ambas ciudades forman parte del conjunto de las 48 ciudades más relevantes de la red urbana nacional (IBGE, 2020). Di Nucci y Linares (2016) también resaltan que la Gran Posadas tiene inserción en un conjunto de centros intermediarios argentinos, componiendo parte de la tercera categoría de importancia de las ciudades mencionadas por los autores.

También es importante considerar la existencia de articulaciones heterárquicas (SPOSITO, 2006; CATELAN, 2013; SPOSITO; CATELAN, 2014), es decir, la potenciación de las articulaciones ya establecidas con los centros de menos importancia y la creación de nuevas relaciones que pueden ser, incluso, transnacionales, dada la ubicación privilegiada de estas regiones.

Pero ¿cómo pueden las tres ciudades cooperar para el desarrollo regional? Esta es la cuestión que nos planteamos, llevando en cuenta la ubicación privilegiada de las ciudades en sus regiones y el alejamiento de los centros metropolitanos de sus países. Nos parece posible suponer que hay una ventana geográfica de oportunidades para el desarrollo regional 
transfronterizo que no puede depender solamente de las políticas de los gobiernos nacionales hechas en las capitales nacionales que están alejadas de este contexto. Es decir, un protagonismo regional puede construirse a partir de la interrelación entre las tres ciudades como motores de sus regiones y, conjuntamente, como un eje tripartito de la gran región transfronteriza.

\section{Horizontes de integración y desarrollo en contextos no metropolitanos sudamericanos}

La región transfronteriza conformada por la provincia argentina de Misiones y por la porción oeste y noroeste de los estados brasileros de Santa Catarina y Rio Grande do Sul respectivamente, ha visto, a lo largo del último siglo, disminuir la intensidad de sus relaciones en la medida de la ampliación de la urbanización, incluyendo el aumento de población y ciudades, si comparamos los movimientos existentes en el período anterior a los conflictos que llevaron a la demarcación de territorios, especialmente las disputas con la República Argentina y la guerra del Contestado en Brasil.

Las interacciones entre Brasil y Argentina han sido tratadas en distintas investigaciones, como las de Ferrari y Dias (2011, p. 160) que destacan la intensidad de los intercambios, incluso en condiciones al margen de la legalidad: "relações comerciais transfronteiriças pelo sistema informal são justificadas pelos habitantes fronteiriços como elementos complementares da organização territorial da fronteira".

Actualmente, la visión de los Estados nacionales sobre el intercambio económico y cultural no corresponde a la intensificación de la ocupación regional y promueve sólo relativamente las políticas direccionadas a su integración ${ }^{6}$. Esta acción de ignorar parcialmente la posibilidad de integración regional tiene repercusión en las economías de ambos países y es responsable por el retroceso de la característica muy común al final de siglo XIX y principios del XX, o sea, de haber nacionales de ambos países moviéndose en la región de manera menos controlada y con más identidad y pertenencia regional, al mismo tiempo que el intercambio lingüístico se mostraba más intenso (FERRARI e DIAS, 2011).

\footnotetext{
${ }^{6}$ Actualmente, por ejemplo, una de las preocupaciones más importantes de los políticos de Santa Catarina, del lado brasileño, es mejorar la fluidez en la frontera argentina para garantizar el ingreso de maíz proveniente del Paraguay para alimentar la cadena productiva agroalimentaria - principalmente utilizado en comida para aves y cerdos. Este circuito productivo dinamiza una amplia red de plantas industriales con variadas actividades económicas tanto directamente vinculadas como en una relación de complementariedad. Por ende, desempeña un rol fundamental en la economía del sur brasileño, sobre todo en la porción cercana a la frontera argentina.
} 
En ese sentido, la idea que proponemos sigue la dirección de evidenciar las posibilidades de integración regional, retomando a la vez características históricas de los movimientos de población antes existentes y avanzando para posicionar las ciudades intermedias como marcos de esta integración, dada la intensa vinculación ciudad-región, especialmente en contexto de urbanización regional (SOJA, 2015). O sea, en este comienzo de siglo, el rol de las ciudades en los procesos de desarrollo económico y social regional es más importante que nunca, porque ellos se configuran como espacios luminosos (Santos; Silveira, 2001) de la producción del modo de vida urbano como totalidad en devenir (LEFEBVRE, 1972).

Mientras que la propuesta de urbanización regional de Soja (2005) está grandemente basada en el contexto de Los Angeles o de otras ciudades regiones globales que tienen fuerte densidad y aglomeran muchos millones de habitantes, estamos movilizando una comprensión de urbanización regional en contexto no metropolitano de baja densidad general y dispersión relativa de población, aunque también pueda abarcar algunos millones de habitantes. En ese contexto, las ciudades intermedias organizan la producción regional, centralizando las decisiones y las relaciones socioculturales, utilizando la infraestructura y las conexiones existentes que podrían ser mejoradas o ampliadas para tornar el conjunto más articulado.

La comprensión de que la ciudad es el elemento que induce las innovaciones y materializa la sinergia del pensamiento colectivo - excepto cuando en contra de lo común en contextos neoliberales contemporáneos (DARDOT; LAVAL, 2017) — forma parte de la amplia obra de Soja (2005, 2015). Siguiendo al autor, consideramos que los centros urbanos más dinámicos pueden aprovechar esta misma potencia para la generación de ideas que apunten a otros caminos, alternativos al ya discutido desarrollo que entiende la economía como único fin en sí mismo.

Ciudades, justicia espacial y las implicaciones en el nivel regional son algunos de los temas tratados por Soja (2014), que apunta posibilidades de pensamiento sobre un derecho a la ciudad regional, es decir, que combina planificación y gestión territorial con un horizonte de articulaciones escalares, tal como la integración entre ciudades que, aunque no sean las más grandes en la escala nacional, tienen acentuada relevancia en sus propios contextos, donde desempeñan roles de centralidad.

Posadas, Passo Fundo y Chapecó son ciudades cuyo desarrollo reciente se debe a reestructuraciones productiva y urbana que les han garantizado inversiones de distintos tipos. Lo que se propone son posibilidades de un desarrollo que se oriente desde demandas locales y 
regionales, de manera interconectada. Soja (2015, p. 377) sugiere casos observados en contextos de países centrales de la economía capitalista en los que, a despecho de las necesidades nacionales o regionales, se ha planeado una urbanización basada en un "regionalismo multiescalar", por su vez produciendo contextos de interacciones transfronterizas intensas.

Más allá de la relativamente densa red de infraestructura viaria, de servicios especializados (salud y educación especialmente) y de diversificación de los centros urbanos, se puede pensar, cuando se observan las dinámicas migratorias pasadas y presentes, los contextos generados en el ámbito de las relaciones supranacionales desde el punto de vista de un desarrollo territorial (BRANDÃO, 2017) que sea más justo y equitativo.

Es posible afirmar que las ciudades son producidas por medio de un conjunto de interacciones entre su contexto regional y la propia capacidad interna de estructurar usos, producciones e intercambios socialmente importantes. Con un análisis sobre la realidad brasileña, Calixto, Oliveira y Soares (2017) nos muestran que las ciudades intermedias son enlaces entre las escalas urbana y regional y cumplen una función de centros nodales de las actividades económicas y políticas, tanto por debajo como arriba en la red de ciudades.

Avanzando a una aproximación con las proposiciones de Brandão (2017), podemos plantear que las ciudades intermedias, sobretodo en escenarios no metropolitanos, deben ser situadas en una lógica horizontal de desarrollo local-regional, en condición de proporcionar equipamientos para uso colectivo público, diversos bienes y servicios.

Promover la integración entre mercados, circuitos y sistemas productivos, instituciones de enseñanza e investigación, medios de comunicación y producción de cultura es lo que se propone como elementos para potenciar la urbanización regional (SOJA, 2015) y, por consiguiente, las interacciones regionales transfronterizas. No se trata de fomentar la competitividad perversa ya señalada por Arroyo (2002), sino la creación de lazos de solidaridad y responsabilidad regional desde los centros urbanos más importantes. Como señala Bitoun (2009) las ciudades tienen un rol importante de responsabilidad territorial a desempeñar frente a su región, sobre todo en contextos de carencia de servicios, infraestructuras y equipamientos.

Planteamos que es necesario encontrar medios para valorar las relaciones regionales a partir de los elementos que pueden ser foco de cooperación y colaboración entre las tres ciudades, con vistas a mejorar la movilidad regional, los flujos turísticos y el intercambio científico y tecnológico para potenciar las características propias de las ciudades y sus 
regiones y avanzar para la superación de cierto retraso en el desarrollo social si las comparamos a otras partes de ambos países. La prevalencia de los roles urbano-regionales sobre la frontera nacional puede ser el medio para alcanzar mejor calidad de vida para las poblaciones.

\section{Conclusiones}

Menos que concluir, lo que podemos hacer es dar direcciones para la reflexión. En este texto hemos buscado reflexionar sobre las posibilidades de la urbanización contemporánea, comprendiendo la centralidad del proceso urbano - no sólo la formación de ciudades, sino, mucho más, la expansión de un modo de vida urbano - en la sociedad desde un análisis bajo la óptica del materialismo histórico-geográfico (SOJA, 1993).

La urbanización, como motor de la vida y producto-condición del capitalismo (HARVEY, 2013), necesita ser apropiada por los sujetos que la producen y, como lo ha sido históricamente, usada como motor del desarrollo direccionado a los intereses comunes (SOJA, 2008).

Al usar la idea de urbanización regional de Soja (2015), no estamos adhiriendo a una perspectiva colonizadora de los espacios regionales por los procesos metropolitanos, sino más bien proponiendo encontrar medios locales para potenciar el desarrollo regional a partir de las características de los centros urbanos. Lo urbano, elevado a región, se constituye desde una articulación multiescalar en ciudades intermedias para superar la demarcación fronteriza de la escala nacional y potenciar interacciones horizontales en los distintos modos de vida regionales.

Sabemos de lo audaz que es esta idea. En un mundo en donde las relaciones han sido cooptadas por la figura del Estado-nación, pensar un desarrollo regional de matriz urbana requiere establecer otra clave de lectura. Significa, de modo profundo, romper lazos históricos y construir una teoría que tenga la potencia no sólo de explicar, sino también transformar las realidades, es decir, comprometerse con el cambio, tal como propone Peet (1982). 


\section{Referencias}

ARROYO, M. Mercosul: discurso de uma nova dimensão do território que encobre antigas falácias. In: SANTOS, M., SOUZA, M. A. A.; SILVEIRA, M. L. Território: globalização e fragmentação. São Paulo: Hucitec; Annablume, 2002. p. 308-314.

BELLET, C.; LLOP, J. M. Miradas a otros espacios urbanos: las ciudades intermedias. Scripta Nova, Barcelona, v. 8, n. 165, 2004.

BELLET, C.; SPOSITO, M. E. B. (Org.). Las ciudades medias o intermedias en un mundo globalizado. Lleida: Edicions de la Universitat de Lleida, 2009.

BITOUN, J. Tipologia das cidades brasileiras e políticas territoriais: pistas para reflexão. In: BITOUN, J.; MIRANDA, L. (Org.). Desenvolvimento e cidades no Brasil: contribuições para o debate sobre as políticas territoriais. Recife: FASE/Observatório das Metrópoles, 2009. p. $17-44$.

BRANDÃO, C. Cidades médias como provedoras de bens e serviços públicos e coletivos e como construtoras de cidadania e de direitos. In: SILVA, W. R.; SPOSITO, M. E. B. (Org.). Perspectivas da urbanização: reestruturação urbana e das cidades. Rio de Janeiro: Consequência, 2017. p. 99-118.

CALIXTO, M. J. M.; OLIVEIRA, H. C. M.; SOARES, B. R. Cidade média e região: notas introdutórias. In: OLIVEIRA, H. C. M.; CALIXTO, M. J. M.; SOARES, B. R. (Org.). Cidades médias e região. São Paulo: Cultura Acadêmica, 2017. p. 11-18.

CATALÃO, I.; MAGRINI, M. A.; LINDO, P. Urbanização, (contra)desenvolvimento e direito à cidade. Boletim de Geografia, Maringá, v. 37, n. 1, p. 199-213, 2019.

CATELAN, M. J. Heterarquia urbana: interações espaciais interescalares e cidades médias. São Paulo: Cultura Acadêmica/Editora UNESP, 2013.

CHESNAIS, F. A mundialização do capital. São Paulo: Xamã, 1996.

CORRÊA, R. L. Construindo o conceito de cidade média. In: SPOSITO, M. E. B. (Org.). Cidades médias: espaços em transição. São Paulo: Expressão Popular, 2007. p. 23-34.

DARDOT, P.; LAVAL, C. Comum: ensaio sobre a revolução no século XXI. São Paulo: Boitempo, 2017.

DI NUCCI, J.; LINARES, S. Urbanización y red urbana argentina: un análisis del período 1991-2010. Journal de Ciencias Sociales, v. 4, n. 7, p. 4-17, 2016.

FERRARI, M.; DIAS, L. C. Territorialidades transfronteiriças na zona da fronteira seca internacional Brasil-Argentina. In: DIAS, L. C.; FERRARI, M. Territorialidades humanas e redes sociais. Florianópolis: Insular, 2011. p. 147-163.

HAESBAERT, R. Regiões transfronteiriças e redes "brasileiras" no Mercosul. In: ENCUENTRO DE GEÓGRAFOS DE AMÉRICA LATINA, 8., 2001. Actas... Santiago: EGAL, 2001. 
HARVEY, D. Ciudades rebeldes: del derecho de la ciudad a la revolución urbana. Madrid: Akal, 2013.

IBGE - INSTITUTO BRASILEIRO DE GEOGRAFIA E ESTATÍSTICA (2020). Regiões de Influência das Cidades 2018. Rio de Janeiro: IBGE, 2020.

LEFEBVRE, H. La revolución urbana. Madrid: Alianza Editorial, 1972.

MABIN, A. Sedimentando a teoria da cidade do Sul no tempo e no lugar. Revista Sociedade e Estado, v. 30, n. 2, p. 323-345, 2015.

MAIDANA, E.; MILLÁN, M. R. Resonancias mediáticas de transformaciones urbanas en Posadas, Misiones. Cuaderno urbano: espacio, cultura, sociedad, v. 8, n. 8, p. 117-136, 2009.

MATIELLO, A. et al. Chapecó/SC: o agronegócio, o setor terciário em expansão e a crescente desigualdade socioespacial. In: SPOSITO, M. E. B.; MAIA, D. S. (Org.). Agentes econômicos e reestruturação urbana e regional: Dourados e Chapecó. São Paulo: Cultura Acadêmica/Editora UNESP, 2016. p. 171-319.

MICHELINI, J.; DAVIES, C. Ciudades intermedias y desarrollo territorial: un análisis exploratorio del caso argentino. Documento de trabajo GEDEUR, n. 5, p. 1-26, 2009.

OSORIO, J. El Estado en el centro de la mundialización: la sociedad civil y el asunto del poder. Ciudad de México: Fondo de Cultura Económica, 2004.

PORTO-GONÇALVES, C. W. A globalização da natureza e a natureza da globalização. Rio de Janeiro: Civilização Brasileira, 2017.

PEET, R. O desenvolvimento da Geografia Radical nos Estados Unidos. In: CHRISTOFOLETTI, A. (Org.). Perspectivas da Geografia. São Paulo: DIFEL, 1982. p. 225254.

SANTOS, M. La naturaleza del espacio: técnica y tiempo, razón y emoción. Barcelona: Editora Ariel, 2000.

SANTOS, M. Técnica, espaço e tempo: globalização e meio técnico-científico-informacional. São Paulo: Edusp, 2013.

SANTOS, M.; SILVEIRA, M. L. O Brasil: território e sociedade no início do século XXI. São Paulo: Record, 2001.

SMITH, N. Desenvolvimento desigual: natureza, capital e a produção do espaço. Rio de Janeiro: Bertrand Brasil, 1988.

SOBARZO, O. Passo Fundo: cidade média com funções comerciais, de serviços e de apoio ao agronegócio. In: SPOSITO, M. E. B.; ELIAS, D.; SOARES, B. R. (Org.). Agentes econômicos e reestruturação urbana e regional: Passo Fundo e Mossoró. São Paulo: Expressão Popular, 2010. p. 29-100.

SOJA, E. Geografias pós-modernas: a reafirmação do espaço na teoria social crítica. Rio de Janeiro: Jorge Zahar Editor, 1993. 
SOJA, E. Algunas consideraciones sobre el concepto de ciudades-región globales. Ekonomiaz, n. 58, p. 44-75, 2005.

SOJA, E. Postmetrópolis: estudios críticos sobre las ciudades y las regiones. Madrid: Traficantes de Sueños, 2008.

SOJA, E. En busca de la justicia espacial. Valencia: Tirant Humanidades, 2014.

SOJA, E. Accentuate the regional. International Journal of Urban and Regional Research, n. 39, p. 372-381, 2015.

SPOSITO, M. E. B. O desafio metodológico da abordagem interescalar no estudo de cidades médias no mundo contemporâneo. Cidades, Presidente Prudente, v. 3, n. 5, p. 143-157, 2006.

SPOSITO, M. E. B.; CATELAN, M. J. Hierarchy and heterarchy in Brazil's urban network. Brazilian Geographical Journal: Geosciences and Humanities research medium, Ituiutaba, v. 5, n. 2, p. 556-574, 2014.

WETTSTEIN, G. Subdesenvolvimento e Geografia. São Paulo: Contexto, 1992. 